\title{
EURIS, a Specification Method for Distributed Interlockings
}

\author{
Fokko van Dijk ${ }^{\S}$ Wan Fokkink* Gea Kolk ${ }^{\S}$ \\ Paul van de Ven ${ }^{\S} \quad$ Bas van Vlijmen ${ }^{\dagger}$ \\ $\S$ Holland Railconsult, PO Box 2855, 3500 GW Utrecht, \\ The Netherlands, $\{f j v a n d i j k, g p k o l k, p h j v a n d e v e n\} @ h r . n l$ \\ ${ }^{\star}$ University of Wales Swansea, Singleton Park, \\ Swansea SA2 8PP, Wales, w.j.fokkink@swan.ac.uk \\ †Utrecht University, Heidelberglaan 8, 3584 CS Utrecht, \\ The Netherlands, vlijmen@phil.uu.nl
}

\begin{abstract}
Safety systems for railways have shifted from electronic relays to more computer-oriented approaches. We present a tutorial on the EURIS method from NS Railinfrabeheer, which champions an objectoriented approach to the specification of interlocking logics. We also give an overview of UniSpec, which is an instance of EURIS for the Dutch railway system.
\end{abstract}

KEY WORDS: interlocking logic; safety critical; distributed system; UniSpec

\section{Introduction}

The control and management of a railway system consists of three separate tasks. First of all, control instructions for the railway yard have to be devised; this is done in what we call the logistic layer. This layer can be fully automated, but in practice it usually consists of an automated system that is controlled by human experts. Second, control instructions to the railway system have to be executed, meaning that they have to be passed 
on to the infrastructure, which consists of points, signals, sections, level crossings, et cetera. This task is almost always fully automated. Third, it has to be guaranteed that the execution of control instructions does not jeopardise safety; that is, collisions and derailments have to be avoided. This is done by means of a so-called interlocking, which is a medium between the infrastructure at the one side, and the logistic layer and its interfaces at the other side. Part of the checks on safety may be performed in the logistic layer, but the ultimate responsibility for safety lies with the interlocking. An interlocking logic is the embodiment of a number safety principles and basic rules, according to which a train moves through a railway yard. It restricts the collection of states for a railway yard. The logistic layer determines the order in which these situations occur, based on efficiency considerations such as convenient connections between corresponding trains.

Traditionally, an interlocking logic served as a local solution for a specific railway yard, whereby the logic could be designed to cope with the peculiarities of the railway yard. Modern computer-based railway systems demand a uniform specification method which can be used to formulate interlocking logics for all railway yards. The restrictions that are imposed by interlocking logics on the states of different railway yards are reasonably consistent, depending mostly on the parameters of the autonomous elements, such as signals and points. Based on this observation, Middelraad cum suis from NS Railinfrabeheer evolved a modular specification method/language EURIS (EUropean Railway Interlocking Specification) [3], to describe fully automated interlocking logics. EURIS assumes an object-oriented architecture, which consists of a collection of generic building blocks, representing the elements in the infrastructure such as signals and points, and of two clearly separated entities in the outside world, representing the logistic layer and the infrastructure. The building blocks, which together make up the interlocking logic, communicate with each other by means of data-structures called telegrams. The building blocks can also exchange telegrams with the two entities that model the logistic layer and the infrastructure. This model can be depicted as follows. 


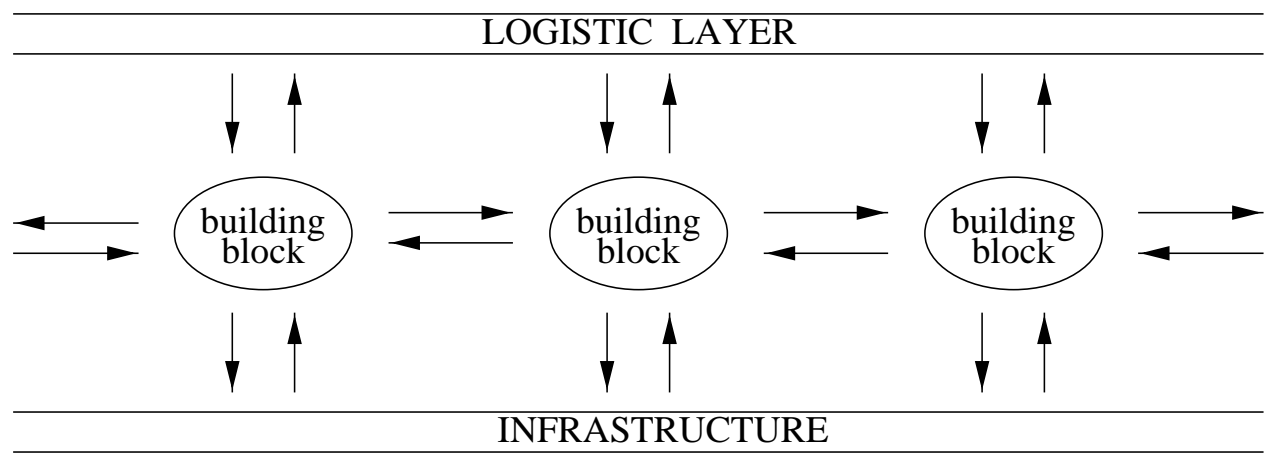

To give an example, suppose that the logistic layer decides that a train should be moved via route $R$. This request is passed on to the interlocking layer, which attempts to claim route $R$; this mission is divided into smaller tasks, which are performed by exchanging telegrams between building blocks. If all building blocks concerned agree that route $R$ can be established without jeopardising safety, then the interlocking reserves this route, after which it passes on the necessary instructions to the infrastructure.

EURIS not only denotes a specification method, it is also the name for a graphically oriented imperative specification language that is based on this method. A so-called Logic and Sequence Chart (LSC) specifies a building block. Each LSC consists of the graphical representation of procedures, which can adapt and test the values of variables, and which can ultimately trigger the transmission of a telegram. Such telegrams can be received by neighbouring building blocks, by the overlying logistic layer, and by the underlying infrastructure. Reversely, each building block can also receive telegrams from neighbouring building blocks, from the logistic layer, and from the infrastructure. The communication channels between the building blocks, the logistic layer, the infrastructure, and the initial values of variables, are recorded outside the LSCs. The graphical format for LSCs evolved as a compact notation when the Nassi Schneidermann diagrams, which were originally used to express EURIS specifications, became unclear due to deep nestings of if-then-else statements.

A strong advantage of a object-oriented architecture is the possibility to reuse components of a specification. In EURIS, the heart of a specification defines the way that different kinds of building blocks handle incoming telegrams. Intuitively, these building blocks represent the separate elements in the infrastructure, such as signals and points. So the main concern of a EURIS specification is to capture the precise behaviour of such elements. As soon as all types of building blocks have been specified in full detail, the 
specification of a particular railway yard is constructed by simply connecting its separate building blocks in the appropriate manner. A second advantage of the distributed approach is that if the behaviour of a certain type of element, say a signal, is changed, then this can be taken into account on the level of EURIS by adapting the specification of the corresponding building block.

UniSpec $[3,11]$ is a particular instance of the EURIS method, which has been developed by NS Railinfrabeheer as a complete set of generic elements to compose interlocking logics for the Dutch railway system. Holland Railconsult has implemented a simulator for UniSpec [9, 10], which enables to animate the behaviour of a UniSpec specification. The simulator is part of a toolset named GUIDE, which is currently used by NS Railinfrabeheer to support both the design and validation of UniSpec specifications. After designing a set of LSCs, the user can join instantiations of these LSCs according to the topology of a railway yard. The result is checked for design rule errors and compiled, after which situations at the railway yard can be simulated via a graphical interface. The simulator enables to locate flaws in an interlocking specification at an early stage of the system engineering process. Furthermore, a simulation session gives a detailed insight of the behaviour of the specification, which can be useful in the communication with customers.

This paper presents a precise and unambiguous description of the working of the EURIS method and its specification language. As for the syntax, we describe the features that are present in $[3,9,10,11,7]$, and that have been singled out as being essential by railway experts. There are several interpretations of the semantics of EURIS around, such as the founding paper [3], and the implementation of the simulator. We formulate an operational semantics for EURIS that, following the simulator, is based on a discrete time model. Bergstra, Fokkink, Mennen, and van Vlijmen [4] presented a semantics for the specification language for EURIS in discrete time process algebra [1].

An extended abstract of this paper appeared as [5].

Acknowledgements This research was funded by Holland Railconsult. Daan van der Meij and Peter Middelraad from NS Railinfrabeheer, and Jan Bergstra, Jan Friso Groote, Marco Hollenberg and Wendi Mennen from Utrecht University, are thanked for their support. 


\section{Overview of EURIS}

In brief, EURIS is a graphically oriented, parallel, event-driven, weakly typed, imperative specification language. A EURIS specification consists of the graphical description of one or more elements, in the form of $L S C s$ (see Section 2.6), which are connected by ports (see Section 2.3). Each element consists of one or more procedures with an imperative character, called flows (see Section 2.2). Elements can send data-structures called telegrams (see Section 2.4) to each other via their ports. Reception of a telegram by a element causes the execution of a flow, which is determined by the telegram and the channel via which the element received the telegram. Changing the value of a variable can also cause the execution of a flow (see Section 2.5).

\subsection{Basic Data Types}

EURIS assumes the two standard data types of Booleans and integers.

- The Booleans consist of 1 , representing true, and 0, representing false.

- The integers are represented by decimal notation. Three standard functions are defined on the integers: addition + , subtraction - , and multiplication $\star$.

In EURIS, Booleans and integers are weakly typed, in the sense that they can be cast to each other. Hereby, the Booleans 1 and 0 are cast to the integers 1 and 0 , and vice versa. Casting an integer other than 1 or 0 to the Booleans produces a run-rime error. Conjunction and negation on the Booleans are captured by multiplication and subtraction on the integers; ' $a$ $a n d ~ b$ ' is given by $a \star b$, and ' not $a$ ' is given by $1-a$. Owing to the functional completeness of conjunction and negation, other logical connectives can also be coded using integer arithmetic.

\section{$2.2 \quad$ Flows}

We consider a certain element, with a unique element name. A flow for this element is a procedure that is built from the following five basic constructs:

- An execution condition formulates under which circumstances the flow is executed. There are two possibilities.

If the execution condition is of the form $\mathrm{T} \triangleright \mathrm{p}$, then the flow is executed if telegram $\mathrm{T}$ is received at port $\mathrm{p}$ of the element; see Sections 2.3 and 2.4 . 
If the execution condition consists of a variable name $X$, then the flow is executed depending on the (change of) value of $X$; see Section 2.5.

- A case tests the value of a variable; the returned value influences the subsequent execution of the flow.

- An assignment adapts the value of a variable.

- A termination statement marks the end of the execution of the flow.

- A send action $\mathrm{p}-\mathrm{T}$ instructs that telegram $\mathrm{T}$ is sent out via port $\mathrm{p}$ of the element. A send action is always followed by termination of the flow.

An element is specified by its flows, where the execution conditions of the flows cover all telegrams that can be received by the ports of the element.

Flows are represented graphically, whereby the tests and assignments of the flow are connected with each other by continuous lines. A test whether variable $X$ equals value $v$ is denoted either by placing $v$ in the flow below variable $X$, or by placing the expression $(X=v)$ in the flow. An assignment of value $v$ to variable $X$ is denoted either by placing $>v$ in the flow below variable $X$, or by placing the expression $(X: v)$ in the flow. The graphical layout of flows is important for the interpretation of tests and assignments; see the picture below.

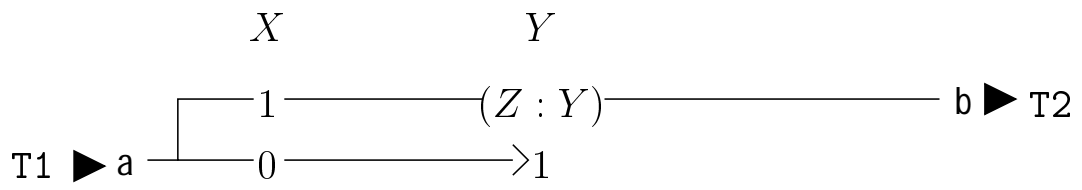

The execution condition at the left of this flow expresses that it is executed if telegram T1 is received via port a. First, the flow tests the value of the variable $X$. If this value is 0 , then the flow assigns the value 1 to variable $Y$, after which it terminates. If the value of $X$ is 1 , then the flow assigns the value of $Y$ to $Z$, after which it sends out telegram T2 via port $\mathrm{b}$. $Z$ is a telegram field; if this field does not yet exist then it is created, and otherwise its value is changed.

\subsection{Ports}

A EURIS specification assumes a logistic layer and an infrastructure, and specifies the behaviour of a number of elements. In particular, it is described how these separate entities send messages called telegrams to each other, and 
how the elements react when they receive a certain telegram from a certain entity. The elements and the logistic layer and the infrastructure can send telegrams to each other via communication channels, which are constructed by the combination of ports. EURIS recognises the following three kinds of ports.

- An element has one or more route ports. Each route port $\mathrm{p}$ of an element e is linked with exactly one route port $\mathrm{p}^{\prime}$ of another element $\mathrm{e}^{\prime}$, establishing a communication channel between e and $\mathrm{e}^{\prime}$. If element e sends a telegram into port $\mathrm{p}$, then this is received by element $\mathrm{e}^{\prime}$ through port $\mathrm{p}^{\prime}$, and vice versa.

- An element may have a port that connects it with the logistic layer, and a port that connects it with the infrastructure. Telegrams can travel from the element to the logistic layer and to the infrastructure via these ports. Vice versa, for each element, the logistic layer and the infrastructure may have a port via which they can send telegrams to this element.

- An element may have two central ports, left and right. Central telegrams with the direction left or right are sent out via the corresponding central port. The destination of the telegram is determined by its so-called central list, which consists of a list of elements, including the current element. Central telegrams that are sent out via left of right are sent to the element to the left or to the right of the current element in the central list, respectively.

\subsection{Telegrams}

A telegram is a data-structure that is composed from several components. Each telegram has a unique name, and carries a so-called telegram table, which consists of variables paired with Boolean or integer values. Intuitively, this table is the record of the telegram; a telegram may be passed on between a number of elements, which all update the information in the table of the telegram. Each element recognises two distinct sets of variables.

1. The set of internal variables for the element is fixed. The names of these variables, together with their initial values, are specified.

2. Telegram variables are of a temporary nature. Their names are the fields of a table of a telegram, and they are only meaningful as long as the flow that belongs to the telegram is being executed. 
If the flow that belongs to a telegram terminates by sending out a telegram, then it attaches the telegram table that was created, or adapted, during the execution of the flow. We distinguish three types of telegrams.

Route Telegrams A route telegram is sent from one entity to another entity, whereby entities can be elements, the logistic layer, or the infrastructure. It consists of a telegram name, a telegram table, and a port name from which it is sent out.

For example, if element e sends out a route telegram with route port name a, then this telegram is sent out via port a. If this port is connected to port $\mathrm{b}$ of element $\mathrm{e}^{\prime}$, then element $\mathrm{e}^{\prime}$ receives this route telegram via port b.

Internal Telegrams An internal telegram is generated inside an element by special types of variables, depending on the (change of) value of such a variable. These variables are explained in detail in Section 2.5. An internal telegram consists of a telegram name together with the name of the variable that produced this telegram, and of an empty telegram table.

Central Telegrams A central telegram is passed on between elements that are on a so-called central list. Each central telegram has a direction, either left of right, which determines in which order it visits the elements on the central list. A central telegram consists of a telegram name, a telegram table, a direction, and a central list.

A central list is a non-empty list $\left[e_{0}, \ldots, e_{n-1}\right]$ of element names. (Multiple occurrences of the same element name in this list are allowed.) If element $e_{i}$ receives a central telegram with this central list, then the flow is executed that corresponds to this central list for element $e_{i}$. The flow tests and adapts values of variables until it terminates, upon which it may send out a central telegram. This new central telegram inherits both the (adapted) telegram table and the central list of the original central telegram. The direction, however, may be reversed. If the emitted central telegram has direction right, then it is passed on to element $e_{i+1}$ in the central list; if the emitted central telegram has direction left, then it is passed on to element $e_{i-1}$. Hereby, $i+1$ and $i-1$ are interpreted modulo $n$ (so $0-1$ results to $n-1$, and $(n-1)+1$ results to 0$)$.

To give an example, consider the central telegram

$$
[\mathrm{T} 1,[(\mathrm{v} 1,15),(\mathrm{b} 1,0)], \text { left },[32,11,7,55,6]]
$$


with name $\mathrm{T} 1$, table $[(\mathrm{v} 1,15),(\mathrm{b} 1,0)]$, direction left, and central list $[32,11,7,55,6]$. Suppose that $\mathrm{T} 1$ has been received by element 32 ; due to the direction of the central telegram its origin must then be element 6 . The execution of the flow that belongs with central telegram T1 in element 32 adapts the telegram table, say to $[(\mathrm{v} 1,-12),(\mathrm{b} 2,1)]$. Let the flow at termination send out a central telegram in the reverse direction, i.e.,

$$
[\mathrm{T} 1,[(\mathrm{v} 1,-12),(\mathrm{b} 2,0)], \text { right },[32,11,7,55,6]]
$$

The destination of this telegram is element 6. (If the direction had not been reversed, then the telegram would have ended up at element 11.)

\subsection{Discrete Time}

In Section 2.4 we distinguished internal variables, which are fixed for an element, from telegram variables. In this section we introduce several types of internal variables. First of all there are input variables, of which the initial values are latched; input variables are distinguished by a version symbol '!'. Then there are internal variables without a version symbol, which are variables of which the value can be adapted without giving rise to the execution of a flow. Finally, there are variables, that, depending on the (change of) value of such a variable, may trigger the execution of a corresponding flow. Such variables carry a version symbol from $\{@, \&, \$, \#$, ?\#, >>\#\}. Before describing these variables in detail, first we say some more about the time domain.

Time plays an important role in the specification of an interlocking logic. It enables to model delays; for example, if a train has passed a section, then for safety reasons this section has to be unoccupied for a certain period of time. We assume a discrete time model, in which time progresses in distinct steps called time slices. The natural numbers can be used to model this time domain: time starts at slice $s_{1}$, and progresses from slice $s_{n}$ to slice $s_{n+1}$. Implementations of interlocking logics usually take the length of a time slice to be exactly 1 second, but it would also be possible to work with a length of say 0.1 second or 10 seconds. Methods such as VPI $[8,6]$ from the General Railway Signal Company and EBS [2] from Siemens, which are used for the implementation of real-life interlocking logics, and the simulator of EURIS, are based on a discrete time domain. Furthermore, it has been shown in practice that it is technically feasible to synchronise the parallel processes of a EURIS specification on time slices. Therefore we hold that a discrete time domain provides a satisfactory simulation of reality, where time progresses 
in a continuous fashion. We consider the different types of variables that may trigger the execution of a telegram.

Cyclic Time-Out $\mathfrak{Q}_{\mathbf{e}, X}(m, n, b)$ represents the cyclic time-out variabele $X$ in element e, with as period the positive integer $m$, with as clock value the non-negative integer $n$, and with the Boolean value $b$. Only the Boolean value of $X$ can be tested, or adapted by an assignment, during the execution of a flow in element e. If $X$ is assigned a Boolean value $b^{\prime}$, then the clock value automatically becomes 0 , so that the new state of the variable $X$ is $\bigodot_{\mathrm{e}, X}\left(m, 0, b^{\prime}\right)$.

Intuitively, the variable $X$ emits a telegram every $n$ time units, if the Boolean value of $X$ is 1 . In other words, if the system evolves into a new time slice, then we can distinguish the following possibilities.

- If the Boolean value of $X$ is 1 , and its clock value $n$ equals 0 , then a telegram is generated, after which the clock value of $X$ is changed to $m-1$.

- If the Boolean value of $X$ is 1 , and its clock value $n$ is greater than 0 , then the clock value of $X$ is changed to $n-1$.

- If the Boolean value of $X$ is 0 , then the clock value of $X$ becomes 0 .

The flow for the telegram that is emitted by $X$ in element e, if its Boolean value is 1 and its clock value is 0 , is specified in the LSC for e.

One-Shot $\quad \&_{\mathrm{e}, X}(b)$ represents the one-shot variabele $X$ in element e, with Boolean value $b$. This value can be tested, or adapted by an assignment, during the execution of a flow in element e.

Intuitively, the variable $X$ emits a telegram exactly once, if the Boolean value of $X$ is changed to 1 . This is modelled as follows. If the system evolves into a new time slice, then:

- if the Boolean value of $X$ is 1 , then a telegram is generated, after which the Boolean value of $X$ is changed to 0 .

The flow for the telegram that is emitted by $X$ in element e, if its Boolean value is 1 , is specified in the LSC for e. 
Toggle $\$_{\mathbf{e}, X}\left(b, b^{\prime}\right)$ represents the toggle variabele $X$ in element e, where $b$ is the Boolean value of $X$ at the end of the last time slice, and $b^{\prime}$ is the current Boolean value of $X$. Only this last Boolean value of $X$ can be tested, or adapted by an assignment, during the execution of a flow in element e.

Intuitively, the variable $X$ emits a telegram if its Boolean values at the end of the last and the current time slice differ. In other words, if the system evolves into a new time slice, then:

- if $b$ and $b^{\prime}$ are distinct, then a telegram is generated, and the value $b$ is changed into the value $b^{\prime}$.

The flow for the telegram that is emitted by $X$ in element e, if $b$ and $b^{\prime}$ differ, is specified in the LSC for e.

Timer $\#_{\mathrm{e}, X}(n, b)$ represents the timer variabele $X$ in element e, with as clock value the non-negative integer $n$, and with the Boolean value $b$. The Boolean value of $X$ can be tested, or adapted by an assignment, during the execution of a flow in element e. The clock value of $X$ can only be tested during the execution of a flow in element e.

Intuitively, the timer $X$ is active if the Boolean value of $X$ is 1 , and otherwise it is inactive with clock value 0 . In other words, if the system evolves into a new time slice, then we can distinguish the following possibilities.

- If the Boolean value of $X$ is 1 , then its clock value is changed to $n+1$.

- If the Boolean value of $X$ is 0 , then its clock value becomes 0 .

Dynamic Time-Out $\quad$ ? $_{\mathrm{e}, X}(n)$ represents the dynamic time-out variabele $X$ in element e, with as clock value the non-negative integer $n$. The clock value of $X$ can be tested, or adapted by an assignment, during the execution of a flow in element e.

Intuitively, the variable $X$ emits a telegram after $n$ time units. In other words, if the system evolves into a new time slice, then we can distinguish the following possibilities.

- If the clock value $n$ of $X$ is 1 , then a telegram is generated, after which the clock value is changed to 0 .

- If the clock value $n$ of $X$ is greater than 1 , and the clock value is changed to $n-1$.

- If the clock value of $X$ is 0 , then the clock value remains 0 . 
The flow for the telegram that is emitted by $X$ in element e, if its clock value is 1 , is specified in the LSC for e.

Parametric Time-Out $\gg \#_{\mathrm{e}, X}(m, n)$ represents the dynamic time-out variabele $X$ in element e, with as period the positive integer $m$, and with as clock value the non-negative integer $n$. The clock value of $X$ can be tested during the execution of a flow in element e. Furthermore, $X$ can be assigned the Boolean value 1 , in which case it is 'set', or the Boolean value 0 , in which case it is 'reset', during the execution of a flow in element e. If $X$ is set, then its clock value is changed to $m$; If $X$ is reset, then its clock value is changed to 0 .

Intuitively, the variable $X$ emits a telegram $m$ time units after it has been set. In other words, if the system evolves into a new time slice, then we can distinguish the following possibilities.

- If the clock value $n$ of $X$ is 1 , then a telegram is generated, after which the clock value is changed to 0 .

- If the clock value $n$ of $X$ is greater than 1 , then the clock value is changed to $n-1$.

- If the clock value of $X$ is 0 , then the clock value remains 0 .

The flow for the telegram that is emitted by $X$ in element e, if its clock value is 1 , is specified in the LSC for e.

\subsection{Logic and Sequence Charts}

An LSC consists of a number of graphical representations of flows. The only extra feature of an LSC is that it allows to combine two flows, in the sense that they may flow together. This means that the tails of these flows are exactly the same, and are shared for the sake of brevity of notation and of efficiency. An LSC takes as basis a list of internal variables, whereby variables carry their version symbols. We give an example.

$X_{1}, X_{2}$ and $X_{3}$ are internal variables. $X_{1}$ is an input variable, which is denoted by the version symbol '!'. $X_{2}$ is a one-shot variable, which is denoted by the version symbol ' $\&$ '. $X_{3}$ does not carry a version symbol. The graphical representations of the flows that make up the LSC are drawn below this list. Thus, we obtain the picture that is displayed in Figure 1. Below each variable name we have drawn an imaginary vertical dashed line. Each test and assignment in a flow is placed on such a dashed line, in order 


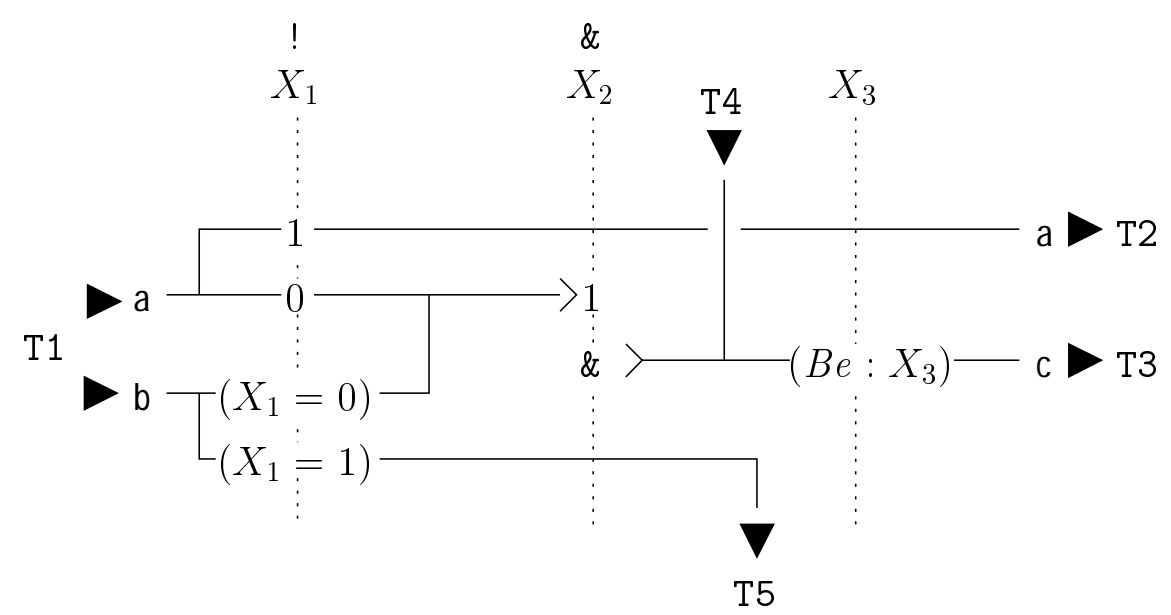

Figure 1: An LSC Example.

to relate it to the variable of which the value is tested or adapted. Note that some of the flows share the same tail, which means that from some point onwards they have the same functionality. However, such flows are independent entities. We explain the meaning of each of the flows.

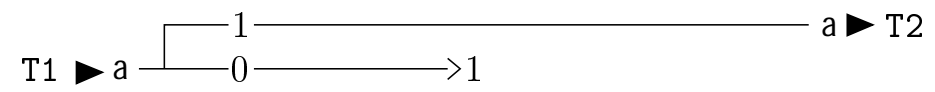

The flow above is executed if the telegram with the name $\mathrm{T} 1$ is received via port a. If the input variable $X_{1}$ is 0 , then this flow assigns the value 1 to the one-shot variable $X_{2}$, after which it terminates. If $X_{1}$ is 1 , then this flow sends out the telegram with the name T2, and with the unaltered telegram table, via port a.

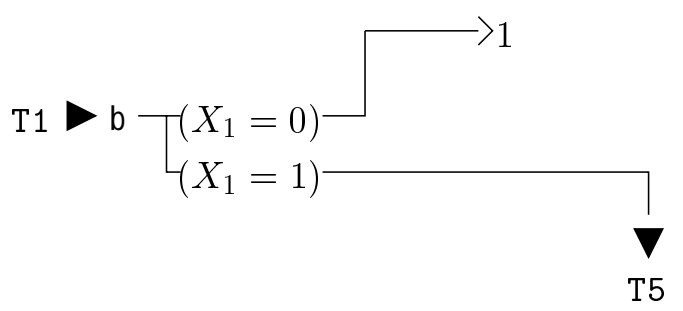

The flow above is executed if the telegram with the name $\mathrm{T} 1$ is received via port $\mathrm{b}$. If the input variable $X_{1}$ is 0 , then this flow assigns the value 1 to the one-shot variable $X_{2}$, after which it terminates. If $X_{1}$ is 1 , then this flow sends out the telegram with the name T5 to the infrastructure. The tests on 
the value of $X_{1}$ in the two examples above are syntactically different, but operationally equivalent.

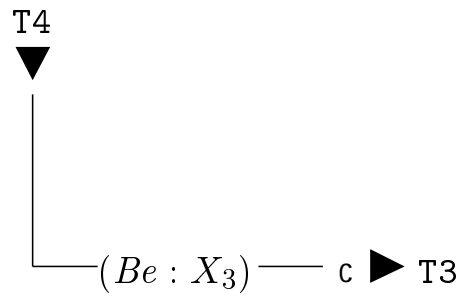

The flow above is executed if the telegram with the name $\mathrm{T} 4$ is received from the logistic layer. It assigns the value of the variable $X_{3}$ to the variable $B e$, denoted by $\left(\mathrm{Be}: X_{3}\right)$. Hereby, $B e$ is a telegram variable, i.e., it refers to a location in the table of the telegram with the name T4. Next, the telegram with the name T3 is sent out via port $\mathrm{c}$.

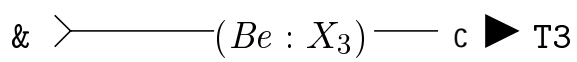

The flow above is generated by the one-shot variable $X_{2}$. It assigns the value of the variable $X_{3}$ to the variable $B e$. Next, the telegram with the name T3 is sent out via port $\mathrm{c}$.

\subsection{Components of an Element}

In the previous section we described how an element is specified by means of an LSC. We explained how the flows in such an LSC can be triggered by either the reception of a telegram by the element, or by a (change of) value of a variable for the element at the end of a time slice. It is a convention in EURIS that the variables are initiated in such a way that the very first flow that is executed for any element is caused by the reception of a telegram; see [10].

We now give a detailed description of the behaviour of an element during a single time step. The behaviour of the full EURIS specification is obtained by putting the behaviours of its elements in parallel. Figure 2.7 depicts the separate components that make up the behaviour of an element, and how they interact. We proceed to explain this picture.

Scheduler An element receives telegrams via its ports at random. Each of these telegrams triggers the execution of a corresponding flow. Since an element cannot execute more than one flow at the same time, it may be necessary to store telegrams in a buffer, where they await execution. The reception and storage of telegrams is performed by the process Scheduler. 


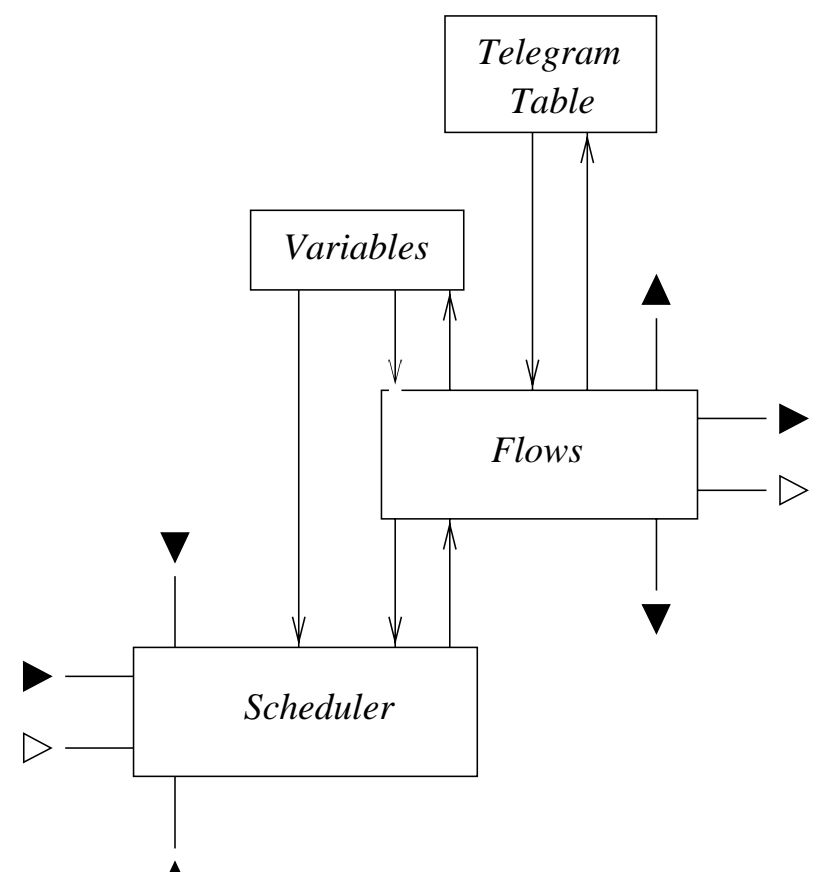

Figure 2: Working of an Element. 
This process also stores internal telegrams that were initiated by (changes of) values of variables at the end of a time slice. The selection strategy of Scheduler is an implementation decision; EURIS does not impose any requirements on this strategy.

If an element is able to start the execution of a flow, then its Scheduler process is instructed to collect a telegram from the buffer, and to pass it on to the process Flows, which executes the corresponding flow. After termination of this execution, Flows instructs Scheduler to select a next telegram from the buffer. In Figure 2.7, at the box Scheduler,

- - and $\triangleright$ represent the route and central telegrams, respectively, that are received by the scheduler from other elements;

- $\boldsymbol{\nabla}$ and $\boldsymbol{\Delta}$ represent the route telegrams that are received by the scheduler from the logistic layer and the infrastructure, respectively.

Flows The Flows process can be instructed to execute a flow. During this execution, values of variables can be tested and adapted. This can be initial variables of the element and variables that are in, or added to, the table of the telegram that is being executed. At termination of the execution of a flow, Flows may send out a telegram through one of the ports of the element. Next, it instructs the process Scheduler to select a next telegram from the buffer. In Figure 2.7, at the box Flows,

- $\longrightarrow$ and $\triangleright$ represent respectively the route and central telegrams to other elements that are sent out by flows;

- $\boldsymbol{\nabla}$ and $\boldsymbol{\Delta}$ represent the route telegrams to respectively the logistic layer and the infrastructure that are sent out by flows.

Variables The Variables process manages the internal variables and of the element. If during the execution of a flow, the value of such a variable is tested or adapted, then this test or assignment is performed by means of a communication between Flows and Variables. At the end of a time slice, (changes of) values of variables may cause that the process Variables produces internal telegrams, which are passed on to the Scheduler.

Telegram Table The Telegram Table process manages the variables in a (temporary) telegram table. If Flows starts the execution of a flow that belongs to a telegram, then it communicates the table of this telegram to Telegram Table. If during the execution of the flow, the value of a variable 
is tested or adapted, or a new variable-value pair is added to the table, then this is performed by means of a communication between Flows and Telegram Table. If at termination of the execution of the flow Flows sends out a telegram, then it obtains the table of this telegram from Telegram Table.

\section{UniSpec}

UniSpec $[3,11]$ is a particular instance of the EURIS method that is under development, with the aim to obtain a complete set of generic elements to compose interlocking logics for the Dutch railway system. It transforms the layout of a railway yard into LSCs in three subsequent steps. First, the topology of the railway yard, and the types of its elements, such as signals and points, are described in a track layout (TL). Moreover, each element is designated a unique name. Next, the elements are provided with ports, and such ports are combined to obtain communication channels between the elements, based on the topology of the track layout. Thus, we obtain the element connection layout (ECL). Next, elements are provided with ports to and from the logistic layer and the infrastructure, and the parameters of the elements and the communication channels are refined, to obtain the logical element connection layout (LECL). Finally, this representation is used to obtain the LSCs of the separate elements. These transformation steps have all been automated in the simulator $[9,10]$.

The syntax of the languages TL and ECL and LECL are based on graphical notations. In the following sections we give brief descriptions of these languages, using a running example.

\subsection{Track Layout}

A track layout (TL) of a railway yard is constructed from its separate elements; we distinguish signals, sections, points, crosses, level crossings, and approach monitoring devices for level crossings. Each element in the track layout has a unique name, based on standard (Dutch) railway conventions. We proceed to present the graphical representations of the elements.

1. A section with name $N$ is represented by

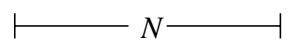




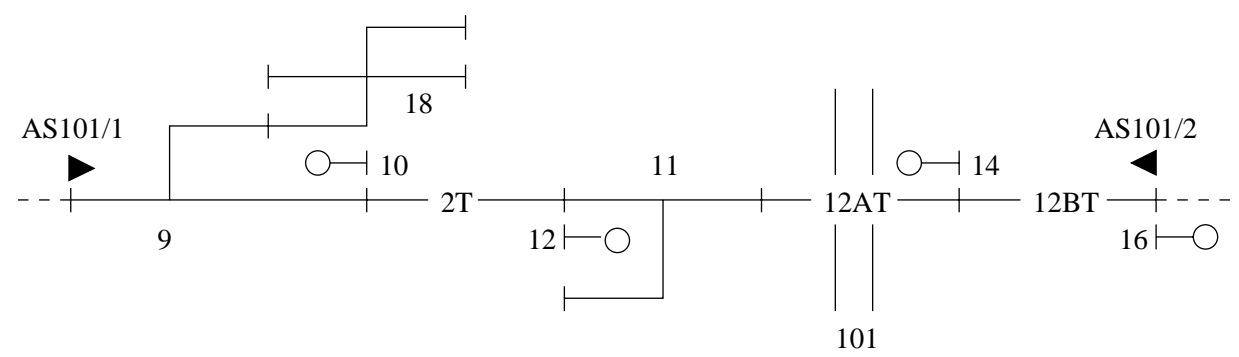

Figure 3: Example of a Track Layout.

2. A signal with name $N$ is represented by

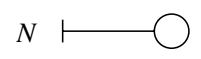

A signal serves for only one direction, which is determined by the orientation of its graphical representation.

3. Points and crosses with name $N$ are represented by
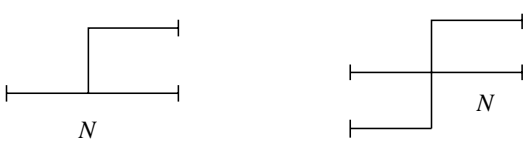

4. A level crossing with name $N$ on a section with name $N^{\prime}$ is represented by

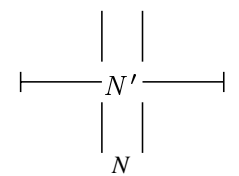

Each path in a railway yard that leads to a level crossing should encounter an approach monitoring device, which is represented by $\stackrel{N^{\prime \prime}}{\longrightarrow}$. The name $N^{\prime \prime}$ always starts with AS, which abbreviates Approach Starting point. An approach monitoring device serves for only one direction, which is determined by the orientation of its graphical representation.

Figure 3 presents an example of a track layout, which is constructed by linking instances of the elements mentioned above. The example is an adaptation of [3, Figure 3]. 


\subsection{Element Connection Layout}

Suppose that we have a track layout. For each element in this layout we generate a graphical element, with a unique name, and with a maximum of four ports called a, b, c en d. We discuss these graphical elements, and the restrictions on names of ports.

1. A signal $N$ that is directed from left to right is represented by the following graphical box, with two ports.

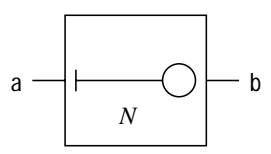

A signal $N$ that is directed in the opposite direction is represented by the symmetric version of this box. In the graphical box of a signal, the port at the bottom of the signal is named a, while the port at the top of the signal is named $b$.

2. Sections, points, and crosses of name $N$ are represented by graphical boxes of the following types, respectively. Again, there are symmetric versions of these elements, which are represented by symmetric versions of the boxes below.

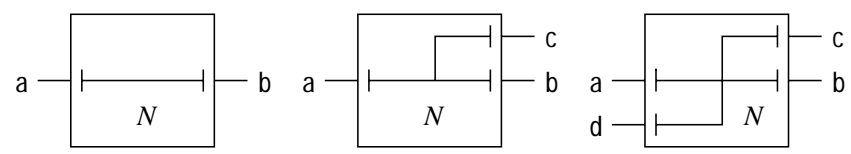

In the box for points, the single exit is named a, while for the double exit at the other side the one at the right is named $b$ and the one at the left is named c. In the box for a cross, the double exit at one side is named a and $d$, while the double exit at the other side is named $b$ and $c$. Hereby, a and b are either both at the left or both at the right.

3. There does not exist an explicit graphical box for a level crossings. They are captured implicitly by monitoring devices: AS (Approach Starting) signals that a train is approaching, while SCD (Signal Clearance Delay) signals that a train has passed the level crossing. AS as well as SCD pass on their information to an element AM (Approach Monitoring). All these elements can monitor trains in only one direction. Since in general trains can move in either direction, a level crossing needs to be protected by at least two AS and SCD elements, and two AM elements are required to monitor approaching trains from op- 


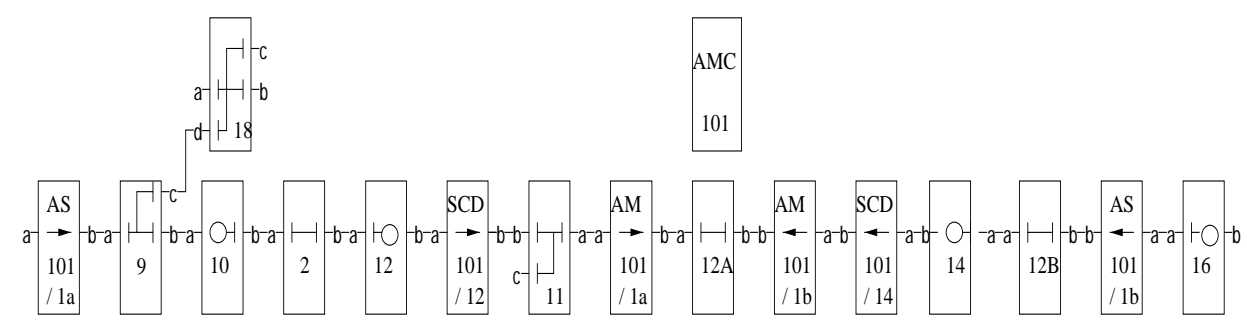

Figure 4: Example of an Element Connection Layout.

posite directions. The information of these two AM elements is passed on to a central element AMC.

Elements AM and AS and SCD of name $N$ are represented graphically as follows.

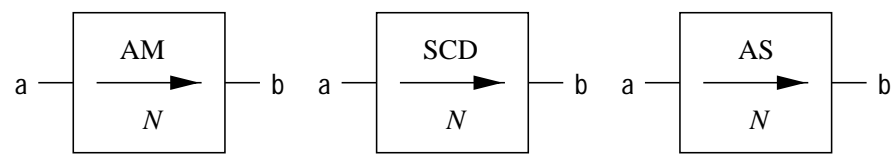

The arrow inside the box designates that the elements monitor trains that travel from left to right. An element that monitors trains in the opposite direction is represented by the symmetric version of its corresponding box.

Elements AMC are represented graphically by boxes without any ports.

In order to transform a track layout into an element connection layout (ECL), all elements are replaced by their boxes. Next, the ports of these boxes are connected to each other, on the basis of the topology of the track layout. Figure 4 depicts how the track layout in Figure 3 is transformed into an element connection layout.

\subsection{Logical Element Connection Layout}

Suppose that we have an element connection layout, which was obtained from a track layout. It is transformed into logical element connection layout (LECL) as follows. Each pair of linked ports produces one or two directed channels between the elements of these ports. The entry side of the channel is marked ' $\mathrm{N}$ ', while the exit side of the channel is marked ' $\mathrm{X}$ '. We distinguish two possibilities. 
1. If one of the ports belongs to a uni-directional element (for AM, SCD or AS), then only one channel is produced, in the same direction as this element. We note that if both ports belong to a uni-directional element, then the transformation rules for TL $\rightarrow$ ECL ensure that both elements have the same direction.

2. If both ports belong to a bi-directional element (for a signal, section, points or cross), then two channels are produced, one for each direction.

An AMC element is connected to each of its AM elements by means of two channels, one for each direction. Furthermore, it is depicted for every element whether there is a channel to or from the logistic layer and the infrastructure. Hereby, the following notations are used.

|: channel from logistic layer to element;

|: channel from element to logistic layer;

I: channel from element to infrastructure;

I: channel from infrastructure to element.

Boxes for signals and points are provided with all four channels. The channels from logistic layer to element and from element to infrastructure carry instructions that a signal should have a certain colour, or that points should move into a certain position. The channels in the opposite direction carry information on the status of a signal or points. Instructions from element to infrastructure are restricted by the requirement that they should not jeopardise safety.

Boxes for sections and crosses are provided with only three of the four channels; the channel from logistic layer to element is missing. The channel from element to infrastructure serves to carry instructions that deal with automatic train protection (ATP), which automatically halts trains that do not respect the speed limit.

Boxes for AS, SCD, and AM do not have any channels. Boxes for AMC have the same three channels as boxes for sections and crosses. The channel from element to infrastructure serves to carry instructions that the level crossing should open or close. 


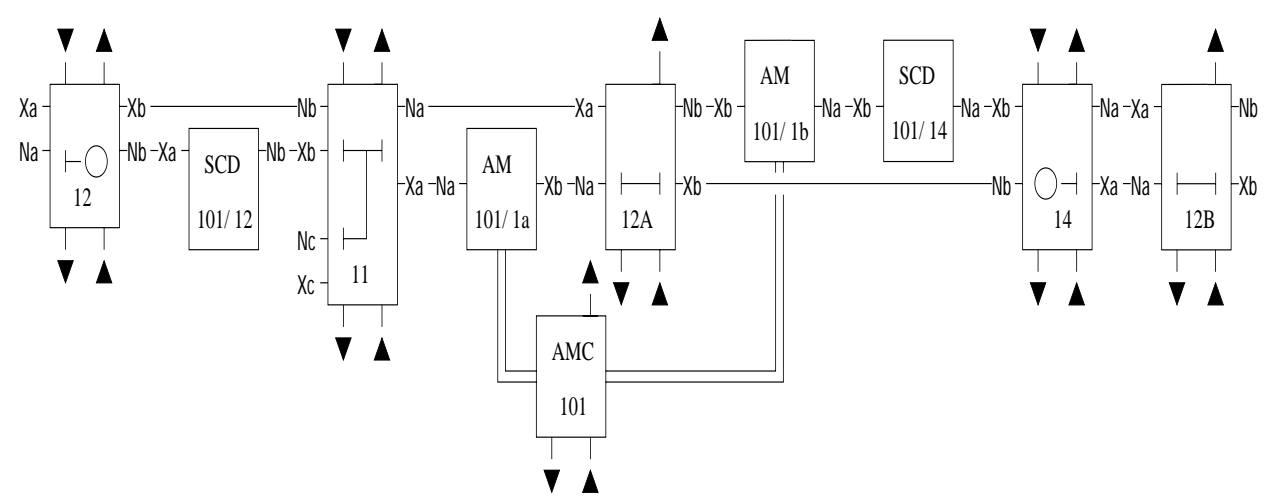

Figure 5: Example of a Logical Element Connection Layout.

An element connection layout is transformed into a logical element connection layout by replacing each element by its corresponding box, and producing directed channels as described above. Furthermore, each box has a private set of internal variables, which are initialised. Figure 5 depicts how part of the element connection layout in Figure 4 is transformed into a logical element connection layout. The initialisation of internal variables is omitted from the picture.

Finally, the logical element connection layout is used to produce an EURIS specification. Each type of box is specified by means of an LSC, which contains the flows that are produced by this box when it receives telegrams. It should be noted that this specification is far from trivial; see for example [3]. Information on channels and the initialisation of variables is not taken into account in LSCs. If a box can be presented in two symmetric ways (as is the case for the box of a signal), then two symmetric LSCs are specified, whereby each flow from left to right in the one LSC occurs as a flow from right to left in the other LSC, and vice versa.

\section{Conclusion}

EURIS is a specification method for interlocking logics. The object-oriented approach of EURIS is a strong point in its favour, and its graphical format allows for compact specifications. However, this compactness can obstruct the clarity of EURIS specifications, especially because in practice EURIS is often treated more like a programming language than as a specification language. LARIS [7], a symbolic variant of EURIS, is a first attempt to remedy this imperfection. 
UniSpec exemplifies the practical use of EURIS for the specification of interlocking logics. Currently, UniSpec contains special operators to cope with specific situations in Dutch railway yards, which hampers the clarity of its semantics. In future, UniSpec should be polished and reduced to a core set of essential operators with sufficient expressive power, and ideally it should be shown to be a correct implementation of the desired interlocking logic. It is yet unclear how functional requirements such as "if points are occupied then their position should be fixed" can be verified for UniSpec. In future interlocking systems, the topology of railway yards may become more dynamic; e.g., positions of signals may become variable. It remains to be seen whether EURIS can cope with such a shift; the specification of elements in UniSpec is based on a fixed topology of railway yards.

\section{References}

[1] J.C.M. Baeten en J.A. Bergstra. Discrete time process algebra. Formal Aspects of Computing, 8(2):188-208, 1996.

[2] J.W.F.M. Beljaars. Prozeß Ablauf Pläne: introduction to a specification method. Report 1992/JBe/3, IB ETS-T\&K, 1992. In Dutch.

[3] J. Berger, P. Middelraad, and A.J. Smith. EURIS, European railway interlocking specification. UIC, Commission 7A/16, May 1992.

[4] J.A. Bergstra, W.J. Fokkink, W.M.T. Mennen, and S.F.M. van Vlijmen. Railway Logic via EURIS. Quaestiones Infinitae XXII, Zeno Institute of Philosophy, 1997.

[5] F.J. van Dijk, W.J. Fokkink, G.P. Kolk, P.H.J van de Ven, and S.F.M. van Vlijmen. EURIS, a specification method for distributed interlockings (extended abstract). In Proceedings 17th Conference on Computer Safety, Reliability and Security (SAFECOMP'98), Heidelberg, Lecture Notes in Computer Science, To appear. Springer-Verlag, 1998.

[6] W.J. Fokkink. Safety criteria for the vital processor interlocking at Hoorn-Kersenboogerd. In Proceedings 5th Conference on Computers in Railways (COMPRAIL'96), Berlin, pp. 101-110. Computational Mechanics Publications, 1996.

[7] J.F. Groote, M. Hollenberg, and S.F.M. van Vlijmen. LARIS 1.0: language for railway interlocking specification. Report, CWI, Amsterdam, To appear, 
[8] J.F. Groote, J.W.C. Koorn, and S.F.M. van Vlijmen. The safety guaranteeing system at station Hoorn-Kersenboogerd. In Proceedings 10th IEEE Conference on Computer Assurance (COMPASS'95), Gaithersburg, pp. 131-150. IEEE, 1995.

[9] F. Makkinga. IDEAL, interlocking design and application language guide and reference. Holland Railconsult, 1994.

[10] F. Makkinga and F. van Dijk. EURIS-simulation tutorial reference. Holland Railconsult, 1995.

[11] D. van der Meij and P. Middelraad. UniSpec. NS Railinfrabeheer, 1996. 